\title{
V2X-Based Mobile Localization in 3D Wireless Sensor Network
}

\author{
Iram Javed $₫$, ${ }^{1}$ Xianlun Tang, ${ }^{1}$ Kamran Shaukat $\mathbb{D}^{2},{ }^{2}$ Muhammed Umer Sarwar, ${ }^{3}$ \\ Talha Mahboob Alam, ${ }^{4}$ Ibrahim A. Hameed $\mathbb{D}^{5}{ }^{5}$ and Muhammad Asim Saleem $\mathbb{D}^{6}$ \\ ${ }^{1}$ School of Computer Science and Technology, Chongqing University of Post and Telecommunication, Chongqing, China \\ ${ }^{2}$ School of Electrical Engineering and Computing, The University of Newcastle, Callaghan, Australia \\ ${ }^{3}$ Department of Computer Science, Government College University, Faisalabad, Pakistan \\ ${ }^{4}$ Department of Computer Science, University of Engineering and Technology, Lahore, Pakistan \\ ${ }^{5}$ Department of ICT and Natural Sciences, Norwegian University of Science and Technology, Trondheim, Norway \\ ${ }^{6}$ School of Information and Software Engineering, University of Electronic Science and Technology, Chengdu, China
}

Correspondence should be addressed to Iram Javed; iram.javed1@hotmail.com and Ibrahim A. Hameed; ibib@ntnu.no

Received 3 December 2020; Revised 11 January 2021; Accepted 27 January 2021; Published 11 February 2021

Academic Editor: Shehzad Chaudhry

Copyright (c) 2021 Iram Javed et al. This is an open access article distributed under the Creative Commons Attribution License, which permits unrestricted use, distribution, and reproduction in any medium, provided the original work is properly cited.

In a wireless sensor network (WSN), node localization is a key requirement for many applications. The concept of mobile anchorbased localization is not a new concept; however, the localization of mobile anchor nodes gains much attention with the advancement in the Internet of Things (IoT) and electronic industry. In this paper, we present a range-free localization algorithm for sensors in a three-dimensional (3D) wireless sensor networks based on flying anchors. The nature of the algorithm is also suitable for vehicle localization as we are using the setup much similar to vehicle-to-infrastructure- (V2I-) based positioning algorithm. A multilayer C-shaped trajectory is chosen for the random walk of mobile anchor nodes equipped with a Global Positioning System (GPS) and broadcasts its location information over the sensing space. The mobile anchor nodes keep transmitting the beacon along with their position information to unknown nodes and select three further anchor nodes to form a triangle. The distance is then computed by the link quality induction against each anchor node that uses the centroid-based formula to compute the localization error. The simulation shows that the average localization error of our proposed system is $1.4 \mathrm{~m}$ with a standard deviation of $1.21 \mathrm{~m}$. The geometrical computation of localization eliminated the use of extra hardware that avoids any direct communication between the sensors and is applicable for all types of network topologies.

\section{Introduction}

The advancement in electronic systems and wireless communication makes wireless sensor networks (WSNs) a great asset for the Internet of Things (IoT). This development provides a way to design a low-power, low-cost, and tiny sensor module, with the ability to process data, sense physical structure, and provide communication within the networks [1-4]. A sensor node consists of a processor, sensing hardware, a transceiver, and a power supply. A node always has limited energy, limited computational power, and memory; thus, a large distributed WSN is required to accomplish a specific task [5]. In WSNs, a lot of sensor nodes operated on a battery are deployed over a sensing region. All of these sensors are used to collect data from other nodes and measure the ecological conditions such as sound, temperature, and vibration. The process data is then forwarded to the base station depending on the system requirements $[6,7]$.

In the past few decades, the use of IoT became more popular, which allows the user to transfer information from many applications. WSNs also gain much popularity in many applications because of their cost-effective nature. Such applications include military applications $[8,9]$, civil process monitoring $[10,11]$, habitat environment monitoring [12-14], health applications [15], home automation $[16,17]$, and vehicular networks [18]. Hence, WSNs provide a new way for proactive computing by getting real-time data from the physical environment. Depending on the system structure and application requirement sensor position is 
required to be known. For this purpose, a sensor node must contain a positioning device [3]. But this is not possible in some scenarios, so a localization system should be able to self-localize and provide an accurate solution without the use of any extra hardware. Localization is a challenging issue for many applications especially for mobile-based WSNs where path planning is the main concern. An extensive research work has been placed for static and $2 \mathrm{D}$ based systems, but none of the algorithms provides an accurate solution for mobile-based WSNs. One de facto solution is the Global Positioning System (GPS), but due to its strict requirement of the line of sight scenario, GPS is not a perfect solution [19]. Furthermore, GPS is not always available and also not working in indoor environment. Furthermore, the cost of GPS receiver is very high, and it consumes more power than a tiny sensor module. Moreover, a mobile node always keeps changing its position so equipping a positioning device does not make it feasible to accomplish its task in a given time span.

A wireless sensor network has four basic components including (1) localized or distributed nodes; (2) a wire/ wireless interconnected network; (3) an information cluster located in a central point; and (4) a set of application systems to process correlation data. Doubtlessly, the main computation is mostly done within the network, because of the large amount of data, algorithms, and techniques implemented within the system. Most of the sensor network applications require measuring the position of the sensor nodes so every system requires an algorithm which is free from extra hardware and communication cost. Furthermore, a localization system should be able to self-localize and calibrate in case of any environmental changes. A lot of WSN localization algorithms are proposed in the literature under range-based and range-free localization categories [1]. It is to be noted that range-based solutions provide high accuracy which use the distance information from the neighbor nodes. A simple example of such a system is triangulation that uses three further nodes to form a triangle. In a rangefree system, the information of absolute distance is not available; instead, the node position is determined through radio connectivity information. Range-free approaches mostly used anchor node deployment for geometrical positioning of the sensor nodes which also provide a low-cost solution. Doubtlessly, each algorithm has its own merits and demerits; its main target is to estimate the node position with accuracy and high efficiency. The flow diagram of the rangebased and range-free localization technique is presented in Figure 1 taken from [20].

The range-based method on the other side used extra hardware that not only increased the cost but also increased the communication and computation overhead. Distance measurement approaches which include time of arrival (ToA) [21], angle of arrival (AoA) [22], time difference of arrival (TDoA) [23], and received signal strength (RSSI) [24] are the best examples of range-based localization algorithms. A brief comparison between range-based and range-free localization algorithms is presented in Table 1.

In the literature, most of the authors have discussed 3Dbased static network localization. However, only a few ideas are available which address the localization of sensor nodes in mobile environment. Therefore, there is a significant need for some automated process to help discover, identify, and locate the mobile nodes within an indoor facility after initial deployment. This motivates us to propose a new localization algorithm consisting of static sensor nodes and flying anchor nodes which helps to locate the position of the sensor nodes even in a changing environment. The self-calibration factor and the use of LQI values make our system more robust and efficient. The rest of the paper is organized as follows. Section 2 discusses the state of the artwork done in WSN localization along with the problem statement and detailed algorithm description. Section 3 presents the simulation results, computational complexity, and lower bound of the proposed localization algorithm. Section 4 concludes the paper with possible future work.

\section{Materials and Methods}

2.1. Related Work. The localization of mobile ad hoc system is very problematic and challenging because it is almost impossible to install an infrastructure with a tiny sensor module especially in a dense environment. Various ad hoc applications also require deploying some anchor nodes with a known position. The aim of this work is to design a localization algorithm based on a mobile anchor node flying on a C-shaped path. To do so, a mobile anchor node always requires some sort of trajectories which is basically not our main consideration for this research. However, a fixed random walk will be considered for simulation purposes. Path planning may be either static or dynamic. In static based path-planning schemes, the execution is taken place before the real process whereas in mobile sensor always follows the predefined path in the entire localization algorithm. In a dynamic path-planning localization, trajectories are not fixed and predefined but particularly drawn according to the environmental conditions.

In a static based path-planning algorithm, the wellknown trajectories are SCAN, DOUBLE SCAN, and HILBERT proposed in [25] and provide the high network coverage. A series of straight lines are proposed in SCAN and DOUBLE SCAN algorithms on which a mobile node is moved in the entire network. In a HILBERT algorithm, a curve is divided into $2 \mathrm{D}$ space and trajectories which are a mixture of vertical and horizontal path. The HILBERT algorithm identifies more beacons during the random walk as compared to SCAN and DOUBLE SCAN trajectories. In another work, two trajectories based on CIRCLE and S-CURVE are proposed [26]. A mobile anchor node is traveling on a circle to cover the entire network. The disadvantage of this scheme is that if the node is not lying in the circle, it may not be localized, and this leads to the network coverage problem. S-CURVE is much similar to SCAN algorithm due to its fixed trajectory and random walk. The structure of $S$ shape also makes it very similar to SCAN algorithm. Furthermore, the authors in S-CURVE algorithm also try to reduce the problem of collinearity during the localization process. In another algorithm, five different trajectories were proposed in [27]. The trajectories 


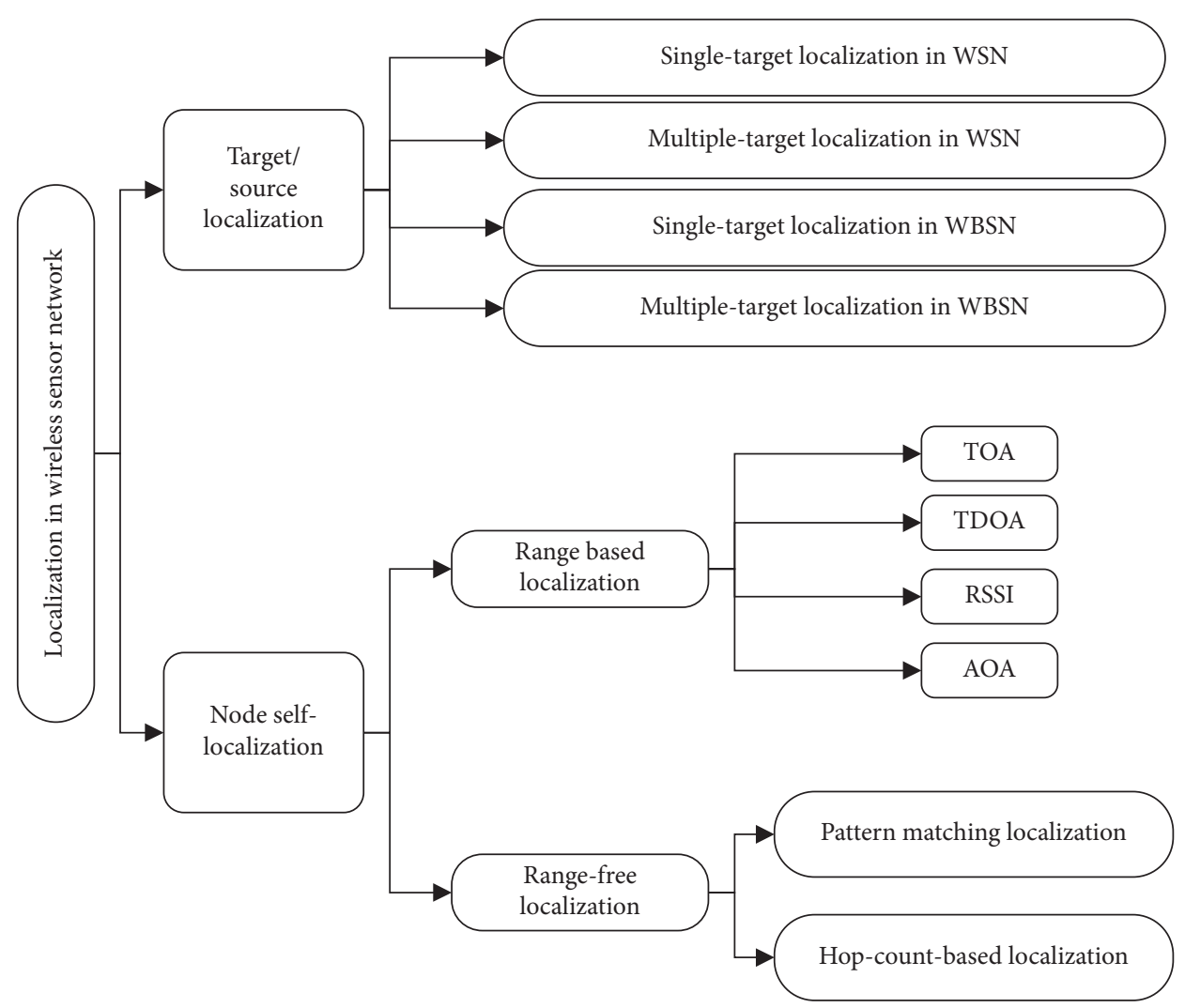

FIGURE 1: A flow diagram of localization algorithms.

TABLE 1: Comparison between range-based and range-free localization algorithm.

\begin{tabular}{lccc}
\hline Algorithm types & Measurement methods & Localization error & Hardware cost \\
\hline \multirow{2}{*}{ Range-based } & AOA & High & High \\
& TDoA & High & High \\
\hline \multirow{2}{*}{ Range-free } & RSSI & Medium & Low \\
& Hop-based techniques & Medium & Medium \\
& Single neighbor & Low & Low \\
& Multineighbor & Low & Low \\
\hline
\end{tabular}

LAYERED-SCAN and LAYEREDCURVE divided the 3D region into different layers. The mobile node travels in the form of SCAN and DOUBLE SCAN format but within the different layered. A variety of other path-planning algorithms were proposed based on dynamic trajectories for the real distribution of WSNs. An undirected graph is generated in [28] based on the artificial intelligence scheme BreadthFirst (BRF) algorithm that transforms mobile walk into the form of spanning tree structure. The movement of the node is dynamic and the mobile node has several different walks that even increase the computation of localization algorithm. The detailed summary of the literature review is presented in Table 2.

2.2. Problem Statement and Assumptions. Let us consider that a set of nodes are deployed on a sensing region where some nodes are static, which are going to localize with the help of mobile anchor nodes. Mobile nodes are the anchor nodes whose position is known with the help of GPS system.
TABle 2: Summary of literature review.

\begin{tabular}{lcccc}
\hline Algorithm & Accuracy & Node density & Traveling speed & Energy \\
\hline$[8]$ & Average & Average & N/A & Low \\
{$[14]$} & Low & Average & High & High \\
{$[25]$} & Average & High & High & High \\
{$[26]$} & Low & High & Low & High \\
{$[27]$} & Average & High & Average & Low \\
{$[28]$} & High & Average & Average & High \\
\hline
\end{tabular}

In brief, a localization process in WSNs will be accomplished in several steps including the computation of distance, reconstruction of sampling distance matrix in presence of noise, and finally computing the localization error using some triangulation techniques.

In a proposed algorithm, we assume that the nodes are deployed on a nonoverlapped network. Let us assume that $N$ number of sensor nodes are deployed on a 3D space and $\boldsymbol{M}$ number of mobile anchor nodes are moving in a C-shaped 
trajectory. The reason to choose C-CURVE trajectory is that most of the network topologies are in this form so we do not fall into a network coverage problem [29]. The nodes have the same communication range as shown in Figure 2.

The system environment includes a fewer number of flying anchor nodes and a number of static sensor nodes deployed randomly. The flying anchor nodes continuously transmit a beacon with location information to unknown nodes in a network to estimate its location. The mobile anchor nodes are moving along a path that is assumed to be predefined. Assuming the uniform distribution of unknown nodes, that is, $\mathbb{R}=(N, M)$,

$$
N=\left\{N_{i}\left(x_{i}, y_{i}, z_{i}\right), \quad \forall N_{i} \in \mathbb{R}\right\},
$$

where $x_{i}, y_{i}, z_{i}$ are the coordinates of the unknown nodes. Similarly, $j$ number of mobile anchor nodes are deploying on a fixed path and transmitting beacons continuously while on a random walk. The received signal strength (RSSI) is measured at each anchor node. The RSSI helps to determine the distance of each node from the anchor nodes and store the distance value in a matrix. The trilateration or triangulation method is then used to form a triangle that helps to measure the localization error by using the centroid formula. We assume that the mobile anchor nodes have enough power in the entire computation of localization error. Furthermore, the communication is in the form of spherical measurement that transforms the $3 \mathrm{D}$ computation in $2 \mathrm{D}$ form and hence makes the system very easy in computation.

$$
\mathbf{M}=\left\{M_{j}\left(x_{i}, y_{i}, z_{i}\right), \quad \forall M_{j} \in \mathbb{R}, M_{j} \longrightarrow \rightarrow\right\},
$$

where $\rightarrow$ denotes the path for mobile anchor node. All the mobile anchor nodes are transferring some beacons in the form of signals. This signal basically includes the localization mobile anchor node. As the computation is in the form of spherical coordinates, so the distance between two unknown nodes is computed by

$$
D_{x_{i}, y_{i}, z_{i}}=N_{i}\left(x_{i}, y_{i}, z_{i}\right)-N_{0} .
$$

Let a node have three beacons from mobile anchor node at a particular location. Then the distance between $N_{1}$ and $N_{0}$ is 1 . Therefore, RSSI also might go down in several points so the exact distance is computed by adding the error factor $\eta$; we have

$$
D_{i}=(x, y, z) \mid\left(r_{i}-\eta_{i}\right)^{2} \leq(x-\hat{x})^{2}+(y-\hat{y})^{2}+(z-\widehat{z})^{2},
$$

where $\hat{x}, \hat{y}, \widehat{z}$ are the estimated position of the sensor node. The distance between mobile anchor node and unknown node is computed by the Euclidean distance formula through equation (5). This is further illustrated in Figure 3.

$$
\sum_{i=1}^{N} d(M, N)=\sqrt{\left(M_{i}-N\right)_{i}^{2}} .
$$

The computation of received signal strength is crucial part of the localization process. We use the RSSI data given

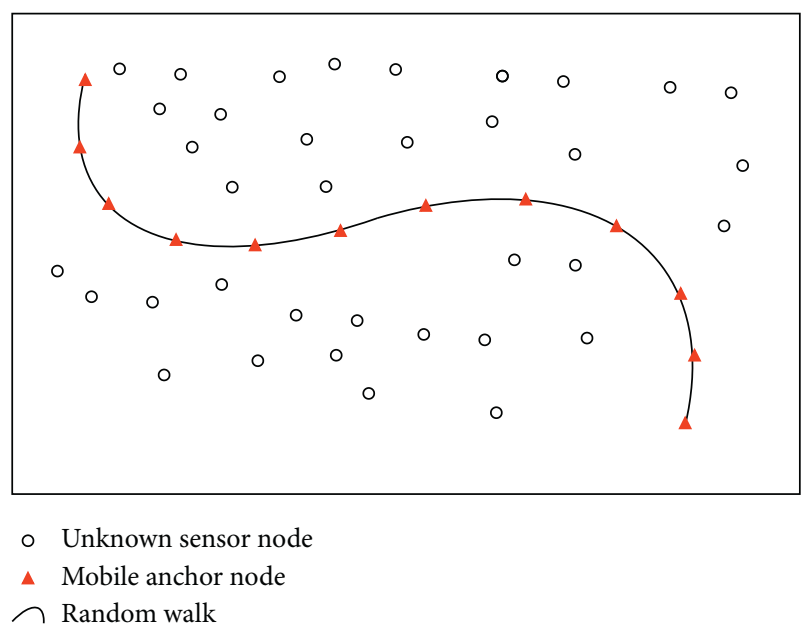

Figure 2: Sensor node deployment and network structure.

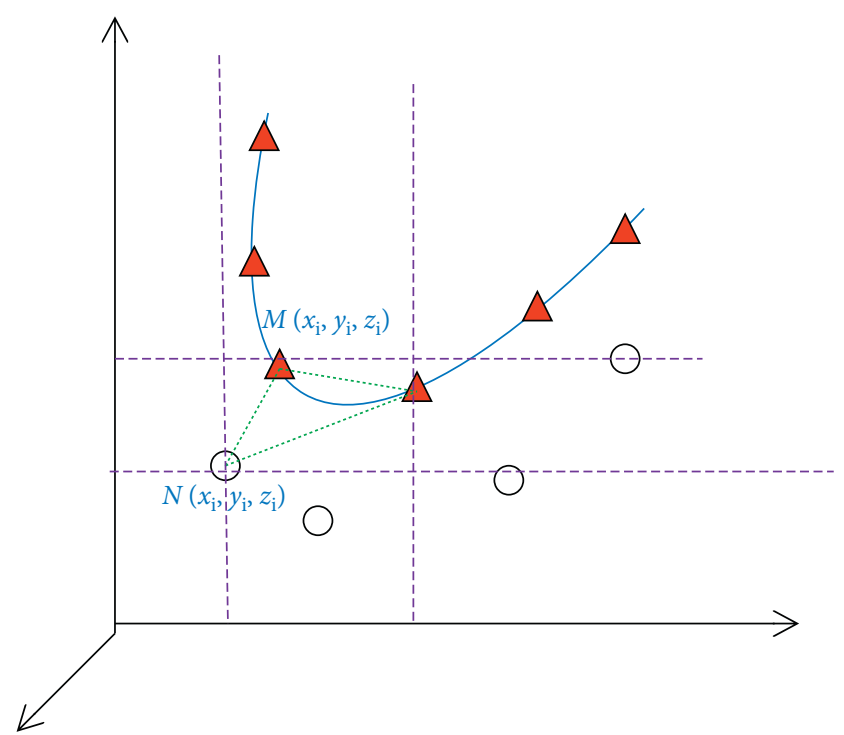

FIGURE 3: Distance measurement between mobile anchor and sensor node.

in GitHub repository [30]. The RSSI is calculated by the following formula [27]:

$$
\operatorname{RSSI}_{i, j}=P_{T}-P_{L_{i, j}}+\aleph_{i, j},
$$

where $P_{T} \mathrm{dBm}$ and $P_{L}$ are a transmission power of device used in measurement phase and pathloss, respectively. Moreover, $\aleph$ represents the noise factor. Equation (3) shows that most power loss occurs at higher frequencies. This means antenna with specified gains; there will be the highest energy transfer in case of lower frequencies. Due to various signal path factors, the loss in the wireless communication path is different from equation (6). By merging the constants, adding losses, and using logarithmic power values, we have

$$
d=d_{0} \cdot 10^{\left(P_{0}-P_{L}+E_{\varphi}\right) / 10 \eta},
$$


where $d_{0}$ is a reference distance corresponding to a reference transmission power $P_{0}$. The pathloss from the signal transmission is derived from [31]; we have

$$
P_{L_{i, j}}=l_{0}-10 n \log _{10}\left(\frac{d_{i, j}}{d_{0}}\right),
$$

where $l_{0}(\mathrm{~dB})$ is the reference pathloss value at $d_{0}=1(\mathrm{~m})$. (1) (m). $N$ is the pathloss exponent value for showing the environment characteristic. $d_{i, j}$ is the distance between nodes $i$ and $j$ in the 3D modeling system.

$$
d_{i, j}=\sqrt{\left(x_{i}-x_{j}\right)^{2}+\left(y_{i}-y_{j}\right)^{2}+\left(z_{i}-z_{j}\right)^{2}} .
$$

The LQI measures the error in the incoming modulation of successfully received packets. It is accessible via the $\mathrm{Mgmt}_{l q i}$ command, whose response gives the LQI values of all neighbors in table form [31]. Similar to RSSI, the LQI has the value range from $\{0 \ldots 255\}$ in which a higher value indicates higher link quality and vice versa. Also similar to RSSI, implementations can differ between manufacturers. Some manufacturers only make use of $7 \mathrm{bits}$, that is, $\{0 \ldots 127\}$. Whereas RSSI provides raw signal strength without caring about link quality, LQI is essentially a measure of data throughput, and its values correlate very well with practical tests of data throughput [31], of which the received signal strength is but one factor. Without incorporating all factors that affect data throughput, for example, interference, noise, and radio characteristics, it is not possible to derive LQI theoretically. However, it is also reasonable to assume that in nonextreme environments, LQI should behave as a function of distance similar to RSSI. The following terms and definitions are used throughout the explanation of the algorithm.

Definition 1. A mobile anchor node is a node that travels over a trajectory with a known coordinate. The coordinates are determined by GPS or some positioning device.

Definition 2. An unknown node is a node whose position is not known and needed to compute by using the anchor node mobility.

Definition 3. A trajectory is a path used for static path planning in the entire network.

The localization scheme requires some basic assumptions.

(1) GPS is attached to all the flying anchors so that the system can identify its real location all the time

(2) In a particular environment, it is also possible that the positioning device provides some inaccuracies

(3) Anchor node is battery operated however equipped with sufficient energy to accomplish the task

(4) Antenna type of anchor is always an isotropic radiator so that it cannot consume much energy
2.3. Proposed Localization Algorithm Based on C-CURVE Trajectory. Assume that the deployment region is in the form of a square and a node is deployed randomly over the sensing region. As the scheme is for range-free algorithms so a region is divided into several networks, that is, $N \omega=\left(N \omega_{1}, N \omega_{2}, \ldots, N \omega_{n}\right)$, in which the networks are not overlapped like a well-known scheme of a point in triangulation (APIT) [32] in which all the nodes are adjacent on overlapped triangles. The overlapped triangles can increase the computation cost as well as energy. The volume of localization is $(1000 \times 1000 \times 1000) \mathrm{m}$, that is, $V m=\left(V m_{1}, V m_{2}, \ldots, V m_{n}\right)$. The distance between the networks is not necessarily computed because the mobile anchor node is moving along the path which is free from the square division. The vertex of the interested region is in the form of minimum and maximum coordinates. The initial coordinates of mobile anchor node are computed by the following formula:

$$
(X, Y, Z)=\left|\frac{\left(x_{\max }-x_{\min }\right),\left(y_{\max }-y_{\min }\right),\left(z_{\max }-z_{\min }\right)}{2}\right| .
$$

The node calculating the initial coordinates of mobile anchor node will act as a reference anchor node. This node always initiates the process in the entire processing of the algorithm. It is also possible that a reference anchor node does not respond due to low battery or node failure. In this case, a control can transfer to the next mobile anchor and select the other three anchor nodes to form a triangulation. This is the beauty of our proposed scheme that the control is transferred to the next level by calibration.

Definition 4. A node initiating the process of localization is said to be a reference anchor node. It may be a static or mobile node.

The entire algorithm can be completed in the following steps.

Step 1.A mobile reference anchor node travels on a static C-CURVE path and broadcasts a beacon node to all other sensor nodes in a communication region. The beacon node contains the ID and coordinates of mobile anchor node.

$$
\tau(t)=\sum R A N D\left(x_{r i}(t), y_{r i}(t), z_{r i}(t)\right) \in \mathbb{R},
$$

where $r$ denotes the communication radius and $t$ is a time factor that denotes the dynamic nature of mobile anchor node in a particular time. $\tau$ is a function that models the trajectory with a random location "RAND."

Step 2. A mobile anchor node then starts estimating distance by sampling RSSI versus distance. At the end of each RSSI measurement, multiple values of RSSI are received because an unknown node keeps sending the RSSI signal until a mobile anchor node is in its communication range. A function of RSSI is formed here 
that is recorded in a matrix form. Here, we introduce a method of link quality induction that is similar to RSSI, but only the successive RSSI introduced by IEEE 802.15.4. The linked quality induction is the highest value of RSSI recorded in the matrix; we have

$$
D_{\text {est }}=\varepsilon(\text { RSSI })=\sum_{i=0}^{3} M_{i}
$$

where $D_{\text {est }}$ and $\varepsilon$ are an estimated distance and mapping function for interpolation of linked quality induction value. In a net phase of RSSI computation, the volume computation is the main problem. At the reference mobile node, we recorded the node coordinates as $x_{0}, y_{0}, z_{0}$ in a square form. Initially, a mobile anchor node is moving from initial coordinates to half of the C-CURVE. According to the nature of the curve, a mobile anchor is moving on a positive radius, that is, $(1 / 2 R)$. Here, a mobile node is needed to make a move to the second half of the curve. This is a tricky part as a mobile node is needed to know the exact position of the trajectory. In the second half of the C-CURVE, the curve side is also changed and given the value of $(-\sqrt{3} / 2)$. The radius is negative that shows the length of the trajectory in a $3 \mathrm{D}$ plane. The random walk length is then computed by

$$
L=\frac{1}{2} R+\frac{-\sqrt{3}}{2}
$$

where $L$ is a localization region. We noticed that the localization region has a problem of network coverage. To overcome this problem, it is possible to change the trajectory in a deep form. For this, we add some constant value on the curve depth that falls over the bounded region. Another solution is to deploy enough anchor nodes on the boundary of the entire network. This also reduces the coverage problem while localizing all the static nodes in a $3 \mathrm{D}$ space.

Step 3. A matrix of all lined quality induction and RSSI values is generated. Now, we need to overcome the interpolation error that is due to having some extra RSSI values from one anchor node towards the sensor node. The compact variations are again generated by mapping the LQI's values from the above matrix.

$$
\varepsilon: \mathrm{RSSI} \longrightarrow=\sum_{i=1}^{N} N_{i} \mathrm{LQI}^{i} \text {. }
$$

Step 4. In the next step, we find the maximum of LQI's values from the LQI matrix; we have

$$
\left[\varepsilon: \mathrm{LQI}_{\mathrm{imax}}\right]=\max \left(\sum_{i=1}^{N} \mathrm{LQI}\right), \quad i \in[1, N] .
$$

To compute the sensor node position, we need all four beacon points. Once we have all four beacon points, two cross sections are constructed. The triangulation is being considered on the circular cross section with their corresponding LQI values.

Step 5. The last step is to implement the centroid-based formula on the matrix of LQI to compute the estimated position of the form

$$
\left[\varepsilon: \mathrm{LQI}_{\mathrm{imax}}\right] \longrightarrow E=\sum_{i=1}^{N} \sqrt{\left(x_{i}-\hat{x}_{i}\right)^{2}+\left(y_{i}-\hat{y}_{i}\right)^{2}+\left(z_{i}-\widehat{z}_{i}\right)^{2}}
$$

where $E$ is a localization error. The pseudocode of our proposed technique is given in Figure 4.

\section{Results and Discussion}

3.1. Simulation Setup. In this section, we can provide a detailed overview of the proposed algorithm. The simulation runs in Matlab and considers an area of $(1000 \times$ $1000 \times 1000) \mathrm{m}$ in $3 \mathrm{D}$ space. The RSSI dataset is being taken from pspachos GitHub [32]. The GitHub data is being emulated in the NEST simulator first to rectify the RSSI signals. After refining the data, we have used these for simulation purposes. The number of mobile anchor nodes was fixed to 5 over 150 unknown sensor nodes. The simulation was run 2000 times to record an initial value of the localization error. The 2000 iterations produce $2000 \times 5$ numbers of anchor nodes. The simulation runs randomly, and the ratio of the localization is determined by the ratio of the number of localizable nodes to the total number of unknown sensor nodes. The localizable nodes are those nodes which are in the sensing range of the mobile anchor nodes. The unlocalized nodes will fall outside the area of network coverage. This can highlight the coverage degree of the path. Hence, the overall degree of a localized node is computed by

$$
E_{\text {Ration }}=\frac{\partial(\longrightarrow N)}{N_{i}},
$$

where $\partial$ is a number of localized nodes from $N$ sensor nodes and $N_{i}$ is a total number of unknown nodes. The simulation is for $3 \mathrm{D}$ area that approximately covers the volume in a cubic meter. Furthermore, the accuracy will be determined by the ratio of localization error and communication radius, that is, $\lambda=E / r$. The accuracy of any localization algorithm can be measured using the standard deviation of unknown nodes. If the standard deviation of the localization error is smaller than the mean error values, which means that the data elements in the form of coordinates are scattered over a $3 \mathrm{D}$ space, this shows that the deployment of sensor node is very crucial in localization scenarios. Furthermore, in case of a mobile-based network, the design of trajectory is very important. Hence, the trajectories are always in a way that covers the entire localization network. The mean localization error is computed by the average and the standard deviation is computed by 


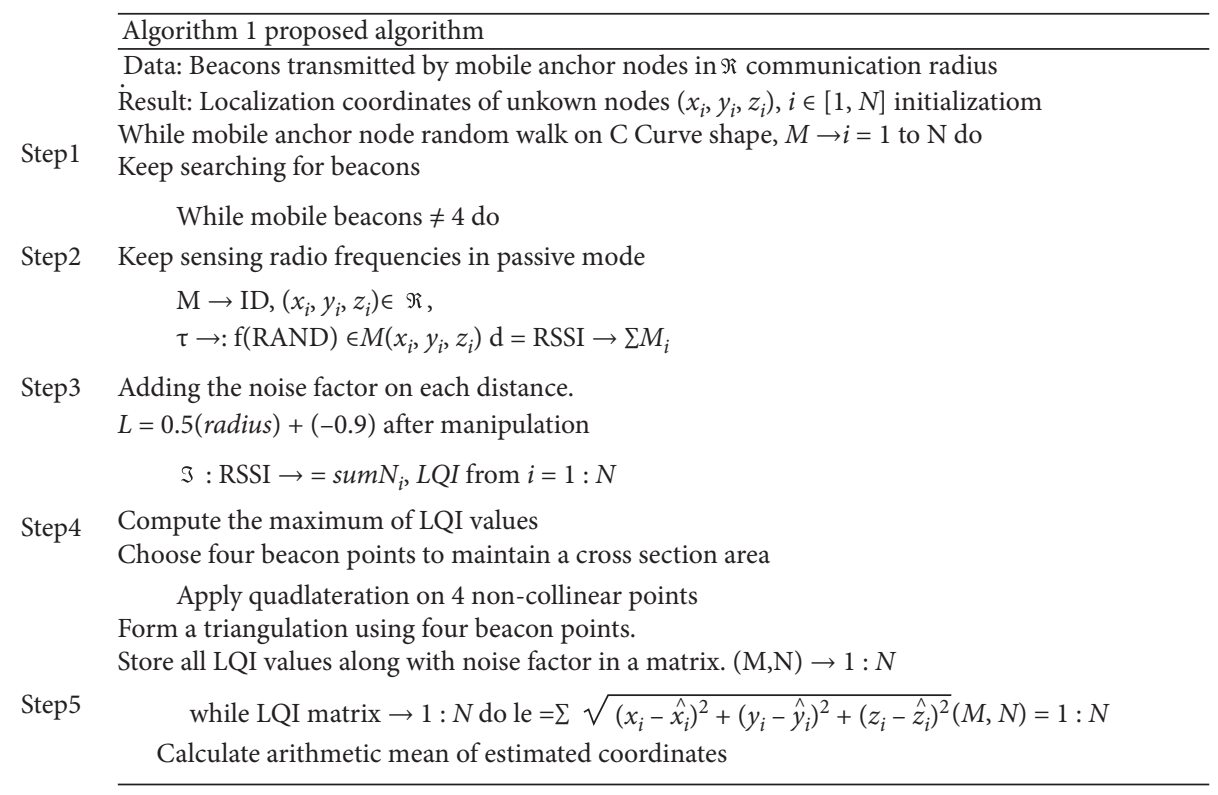

FiguRE 4: C-CURVE-based proposed algorithm.

$$
\begin{aligned}
& \mu E=\frac{1}{\partial} \sum_{i=1}^{\partial} \lambda, \\
& \sigma E=\frac{1}{\partial} \sum_{i=1}^{\partial}(\lambda-\mu)^{2} .
\end{aligned}
$$

In our proposed algorithm of C-CURVE, the localizable points are determined on the basis of the number of successive LQI values. The successive LQIs are the countermeasure upon all the RSSI values. Therefore, the number of successive LQIs is the best approach to find the number of localized nodes. The path in our proposed scheme is in the form of a dual curve that almost covers the entire region of the network. However, some nodes at the edge of the graph might not listen to the mobile anchor call, which may produce some errors in the localization process. We found that the nodes closed to the paths are giving very nominal errors and due to having a curvy shape, most of the nodes are in the neighbor of the mobile path. This reflects the higher accuracy of the proposed system. Nodes within the sensing region also send multiple RSSI values until a mobile anchor is in the domain of the sensing region. However, most of the RSSI fail due to low battery and multipath effect. This is another reason for obtaining an LQI value instead of RSSI. As mentioned earlier, this work is based on simulation only, so we use the GitHub database for RSSI and consider the higher RSSI as an LQI from a node to mobile anchor. The following parameters are used for the initial simulation shown in Table 3.

3.2. Localization Error. In a $3 \mathrm{D}$ environment, each node has a localization cost and error; hence, in case of 3D system, we are interested in recording the average localization error. The mean localization error is recorded as $1.4 \mathrm{~m}$, which is far better than SCAN, DOUBLE SCAN, and HILBERT
TABLE 3: Simulation parameters.

\begin{tabular}{lc}
\hline Parameter & Range \\
\hline Area & $(1000 \times 1000 \times 1000) \mathrm{m}$ \\
Mobile anchor speed & $8 \mathrm{~m} / \mathrm{sec}$ \\
Number of mobile anchor nodes & 5 \\
Number of unknown nodes & 150 \\
Communication radius & $(100-150) \mathrm{m}$ \\
Path loss exponent & $2-5$ \\
\hline
\end{tabular}

algorithm. The mobile anchor node, true location of sensor, and estimated position are shown in Figure 5.

Considering that a node $N_{1}$ is close to the mobile anchor node at a certain period of time, the following RSSI were recorded. The LQI is then measured using the IEEE Mgmt request command as shown in Table 4.

The average localization error is computed along the entire axis with different communication radius. The number of mobile anchor nodes was fixed to check the authenticity of the algorithm for different radius. It is to be observed that the number of localization points is not decreasing but the weaker RSSI can affect the error for higher communication radius as shown in Figure 6 and the graph plot is shown in Figure 7. RSSI and LQI values represent the power level gain after loss in cable and at antenna. The higher RSSI values basically represent the stronger signal which is always required to form a triangulation between several sensor nodes. If the signal is weaker, the node cannot communicate with other nodes efficiently. Hence, it can be through a value that is beyond the boundary of the considered triangle. All the studies related to RSSI-based localization have difficulties to establish a link between signal strength and distance. In real deployment, it is challenging to map RSSI to the distance, and obtaining a fading function reflects that RSSI is affected by pathloss, shadowing, and fading, which also affect communication radius. 


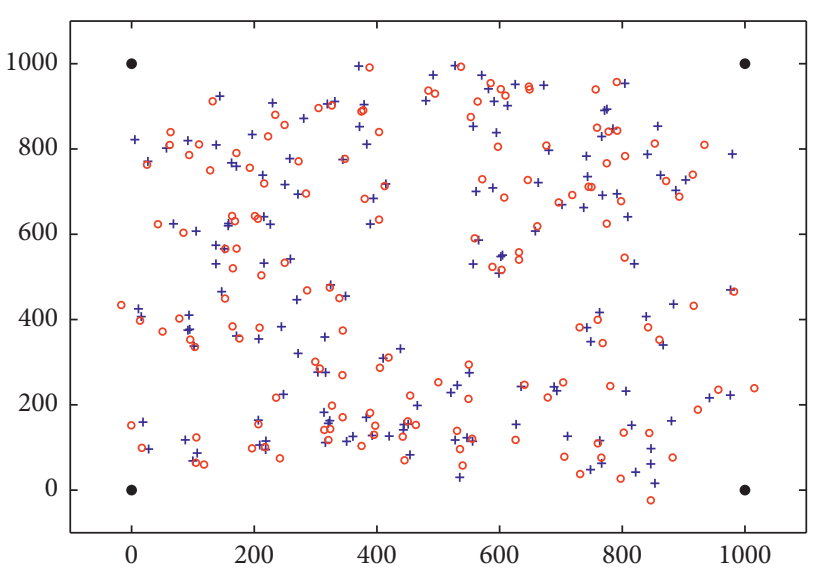

- Mobile anchor locations

+ True location

- Estimated location

Figure 5: Position of mobile anchor, sensor node, and estimated node position.

TABLE 4: Sample LQI measurement.

\begin{tabular}{|c|c|c|c|}
\hline Node & $\begin{array}{l}\text { RSSI in upward } \\
\text { direction }\end{array}$ & $\begin{array}{l}\text { RSSI in downward } \\
\text { direction }\end{array}$ & $\begin{array}{l}\text { Successive } \\
\text { LQI }\end{array}$ \\
\hline N1 & 0.967319090984647 & 0.253562174262323 & $\begin{array}{l}\text { Mgmt LQI } \\
\text { request }\end{array}$ \\
\hline N2 & -0.958798243408558 & 0.284087888577925 & $\begin{array}{l}\text { Mgmt LQI } \\
\text { request }\end{array}$ \\
\hline N3 & 0.524669344883845 & -0.851306101551702 & $\begin{array}{l}\text { Mgmt LQI } \\
\text { request }\end{array}$ \\
\hline N4 & -0.478708185913592 & -0.877974072931153 & $\begin{array}{l}\text { Mgmt LQI } \\
\text { request }\end{array}$ \\
\hline
\end{tabular}

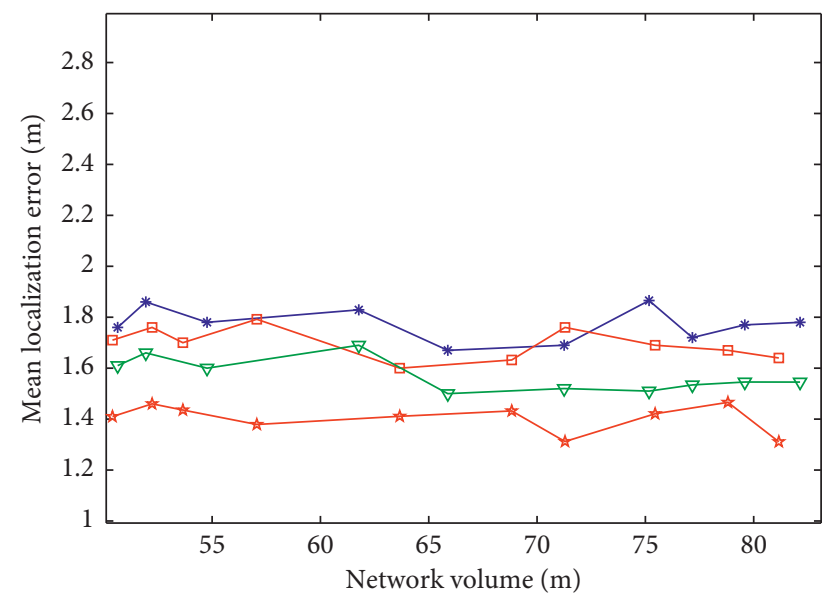

* $r=150 \mathrm{~m}$, anchor node $=5$
$\square \quad r=140 \mathrm{~m}$, anchor node $=5$
$\square \quad r=130 \mathrm{~m}$, anchor node $=5$
ॠ $\quad r=110 \mathrm{~m}$, anchor node $=5$

FIGURE 6: Average localization error in different region of the network with 5 anchor nodes.

3.3. Computation of Node Mobility and Network Coverage. The node mobility is always measured by the initial position and the relative change in the location of mobile anchor

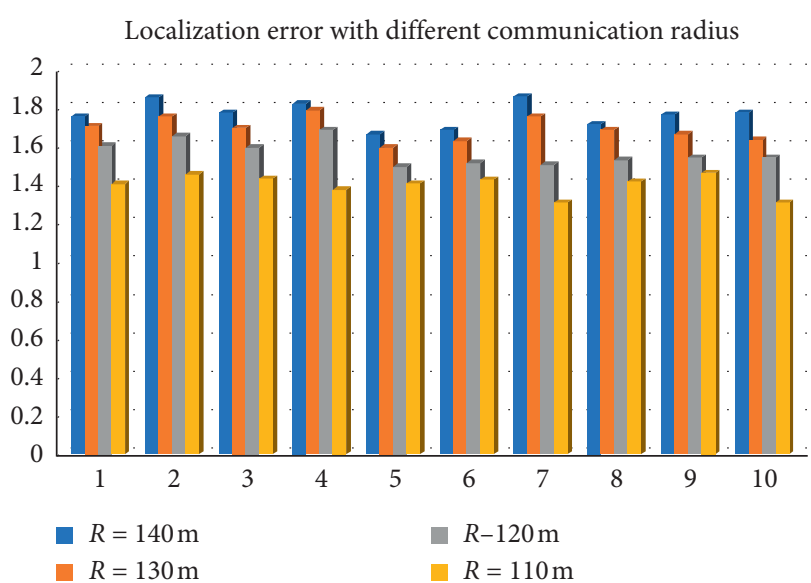

Figure 7: Localization error estimation with different network coverage.

nodes. With the concept of speed and velocity, the mobility of the mobile anchor node is measured along with the specific time. The addition of time variation subtracting with the average distance to the initial point is known as mobile node mobility; we have

$$
M_{i}=\frac{1}{T-\Delta}
$$

The total sum of distance upon time is multiplied to initial mobility factor to compute the final movement of mobile anchor node.

$$
\begin{aligned}
M_{f} & =\prod_{i=1}^{M} \sum_{t=0}^{T-\Delta t}\left|D_{i}(t+\Delta t)-D_{i}(t)\right| \\
D_{i}(t) & =\left(\frac{1}{n-1}\right) \prod_{i=1}^{M} \sum_{t=0}^{T-\Delta t}\left|D_{i}(t+\Delta t)-D_{i}(t)\right|-\sum D(N, M),
\end{aligned}
$$

where $T$ is a periodic motion for each mobile anchor and $\Delta t$ is a change in time for each mobile node so that there should not be any collision between the nodes. Hence, the final mobility is measured by equations (5) and (22). We have

$$
D_{i}(t)=\left(\frac{1}{n-1}\right) \prod_{i=1}^{M} \sum M_{i} \text {. }
$$

The probability density function (PDF) can be used to define the accuracy of the C-CURVE method. To compute the PDF, the Rayleigh fading is considered to measure the localization error. The form of the Rayleigh is shown in Figure 8. In presence of Rayleigh fading with obstructions and reflection, the error might be affected because of multipath fading [33]. Another factor of time constant is associated with variations along with the mobile node movement in seconds or even in minutes. The effect of the carrier also plays an important role with $f c=900 \mathrm{MHz}$ or approximately $1.9 \mathrm{GHz}$ for the mobile node. The effect of localization error is further shown in Figure 9. 


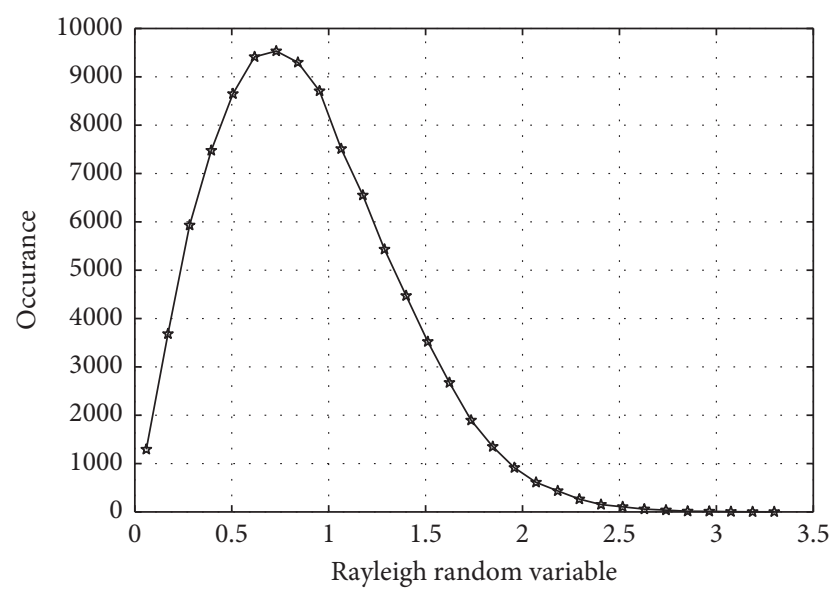

Figure 8: Rayleigh fading.

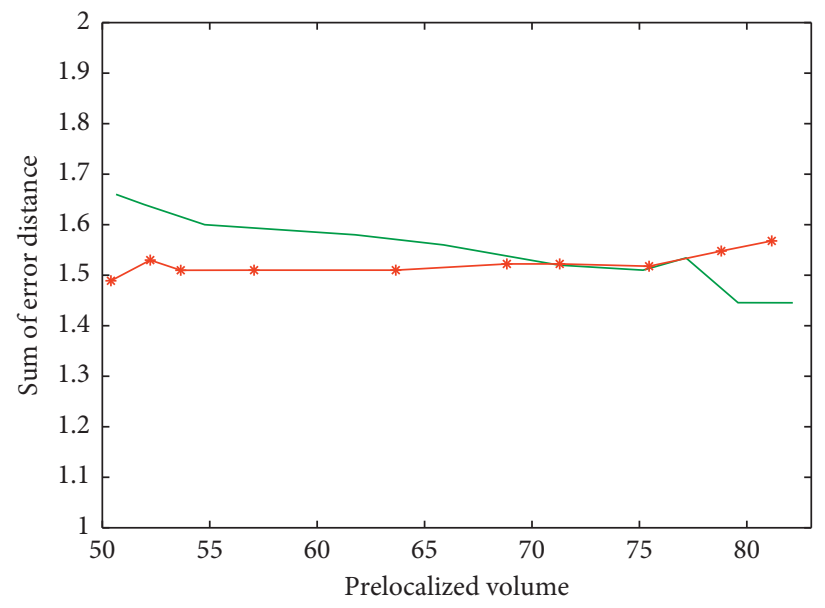

FIGURE 9: Localization error in presence of noise.

From Figure 9, we observed that the localization error is gradually increased in the presence of fading. The amount of data elements spreading along the region is very nominal. And it is possible to retain the correct error by using the framework like extended Kalman filtering and fuzzy logic [34]. The network lifetime and coverage in our proposed system are well described.

3.4. Comparison with Existing Techniques. To authenticate our proposed solution, we have compared our localization algorithm with the state-of-the-art localization techniques based on mobile anchor-based localization. The well-known methods SCAN, DOUBLE SCAN, and HILBERT are being compared with our C-CURVE localization algorithm. SCAN trajectories are very simple to compute with the path on some straight lines with parallel distribution along $y$-axis. However, in a round movement, the mobile anchor node lost enough energy in a given radius $R$. The distance in a SCAN is computed by the following formula:

$$
D=\left(\frac{L}{R}+1\right) \times L+\left(\frac{L}{R}\right) \times R=\left(\frac{L}{R}+2\right),
$$

whereas for the DOUBLE SCAN and HILBERT algorithm, the distance is computed by the following relationships:

$$
\begin{aligned}
& D=2\left[\left(\frac{L-R}{2 R}+1\right)\right] \times L+\left(\frac{L-R}{2 R}\right) \times 2 R, \\
& D=4^{n} \times R=\left(\frac{L}{R}\right)^{2} \times R=\frac{L^{2}}{R} .
\end{aligned}
$$

The SCAN, DOUBLE SCAN, and HILBERT are simulated on the area of $(450 \times 450) \mathrm{m}$, whereas we simulate our idea for $(1000 \times 1000 \times 1000) \mathrm{m} 3 \mathrm{~d}$ space. Hence, the suitability of our proposed system is far better than other techniques. We observed that the localization error is still more than $1.2 \mathrm{~m}$ for a small region. SCAN and DOUBLE SCAN have almost the same localization error with a difference of $0.02 \%$, whereas in HILBERT, this percentage is increased to $3.5 \%$. The only reason for this variation is a 


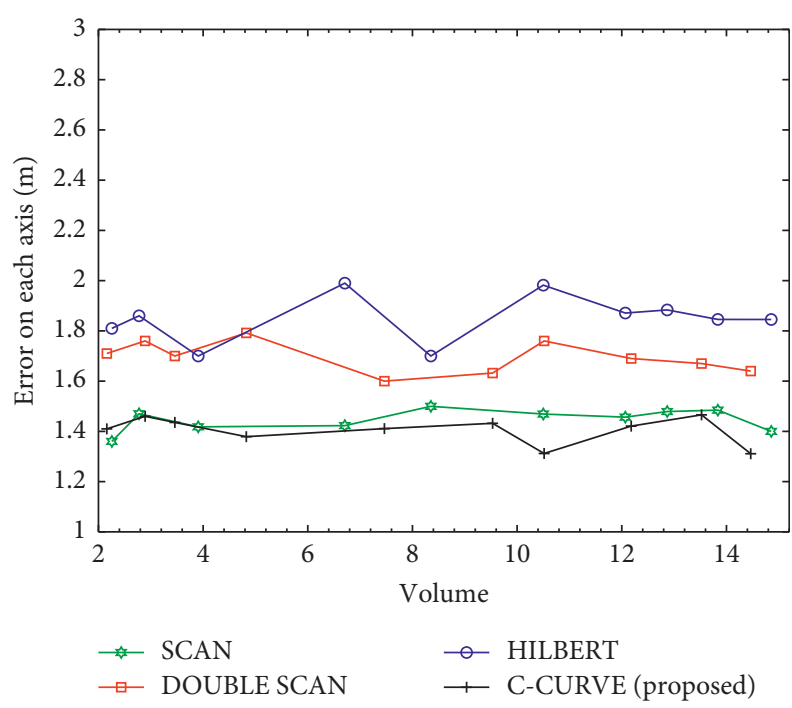

(a)

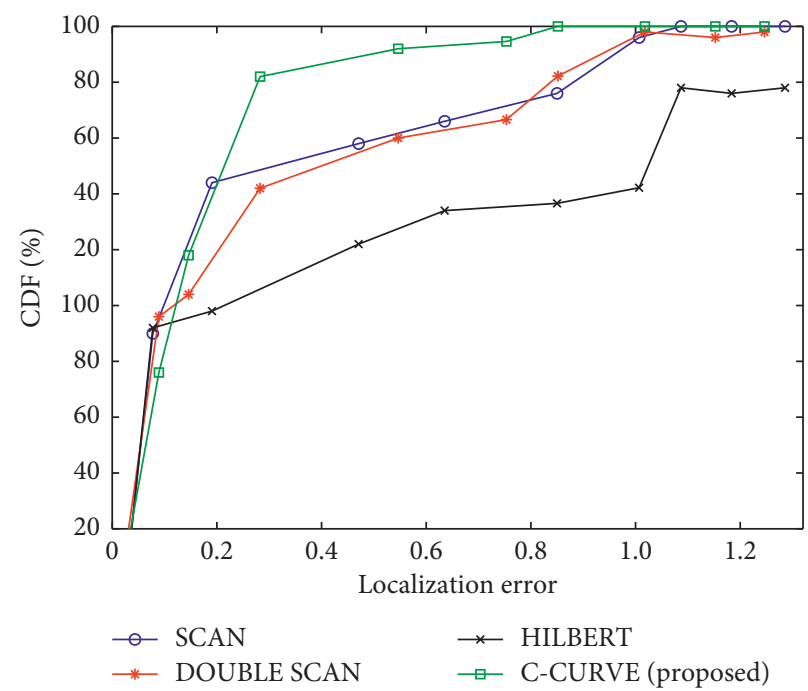

(b)

Figure 10: (a) Comparison of SCAN, DOUBLE SCAN, and HILBERT algorithm with our proposed solution (20 m with $20 \%$ anchor density). (b) CDF of localization error.

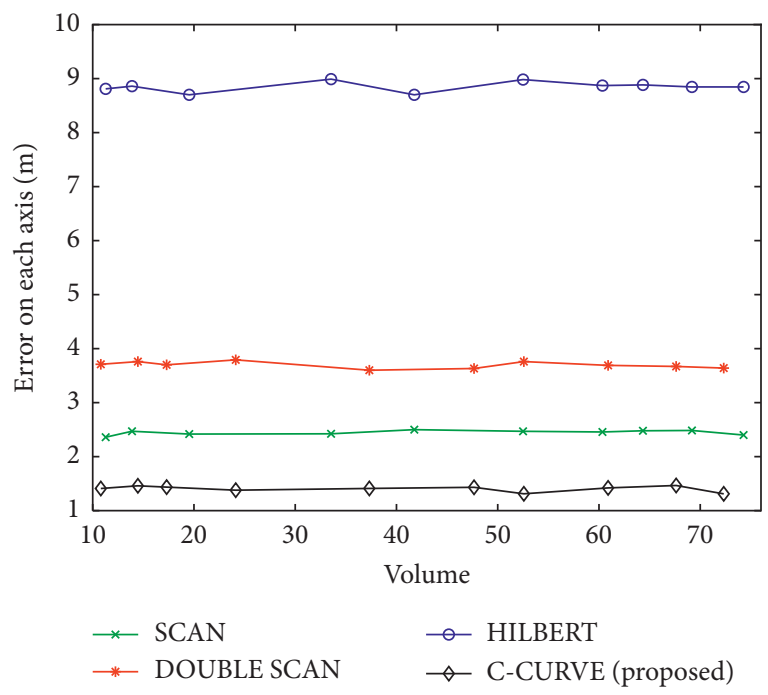

(a)

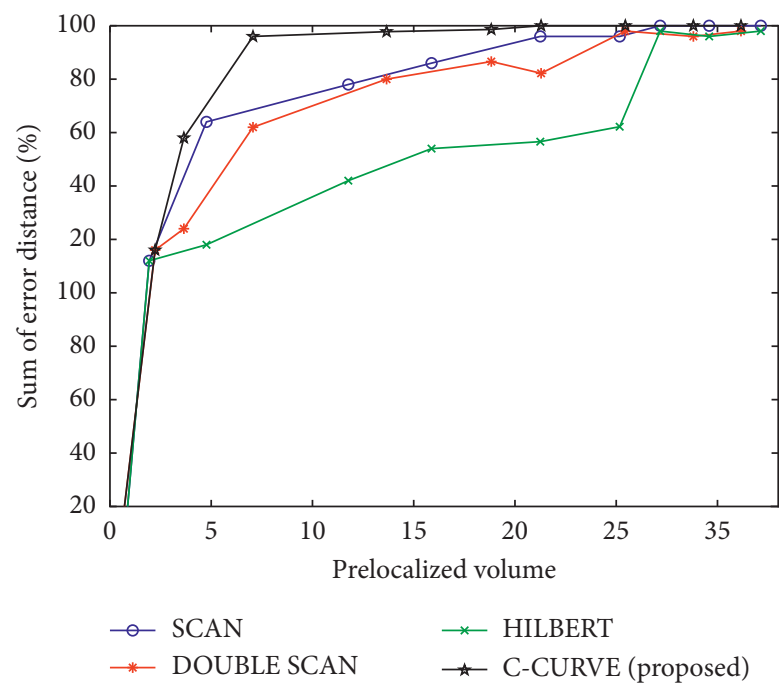

(b)

FIgUre 11: (a) Comparison of SCAN, DOUBLE SCAN, and HILBERT algorithm with our proposed solution (70 m with 50\% anchor density). (b) CDF of localization error.

small variation in anchor node density and a very small region of $30 \mathrm{~m}$. Furthermore, there are a lot of turns in the HILBERT algorithm that increase the complexity, whereas in our proposed system, there is a very slight movement of mobile anchor along its trajectory, which helps to save enough energy and hence computation cost. The comparison analysis is shown in Figure 10(a) along with the CDF for small distance as shown in Figure 10(b).

We observe that the CDF for DOUBLE SCAN was better than that for SCAN and HILBERT algorithm. However, after $5 \mathrm{~m}$, it starts gaining worse localization error due to not having enough beacon on the trajectory part. It was observed that the localization error of SCAN, DOUBLE SCAN, and
HILBERT becomes too worse in case of high resolution. Even just for a $60 \mathrm{~m}$ distance, the error becomes $(2.4,3.9,8.7)$ $\mathrm{m}$, respectively. The C-CURVE algorithm is not affected because of having enough network coverage path on the network. That is why our system gives a highly precise localization solution for mobile anchor-based localization system. By increasing such distance only up to $\sim 70 \mathrm{~m}$, the error goes high as shown in Figure 11 along with $\mathrm{CDF}$ for the particular distance.

The proposed algorithm has been compared with some other techniques based on mobile anchor flying over a random path. The mobile beacon assisted localization scheme presented in [35], used a movement strategy of 


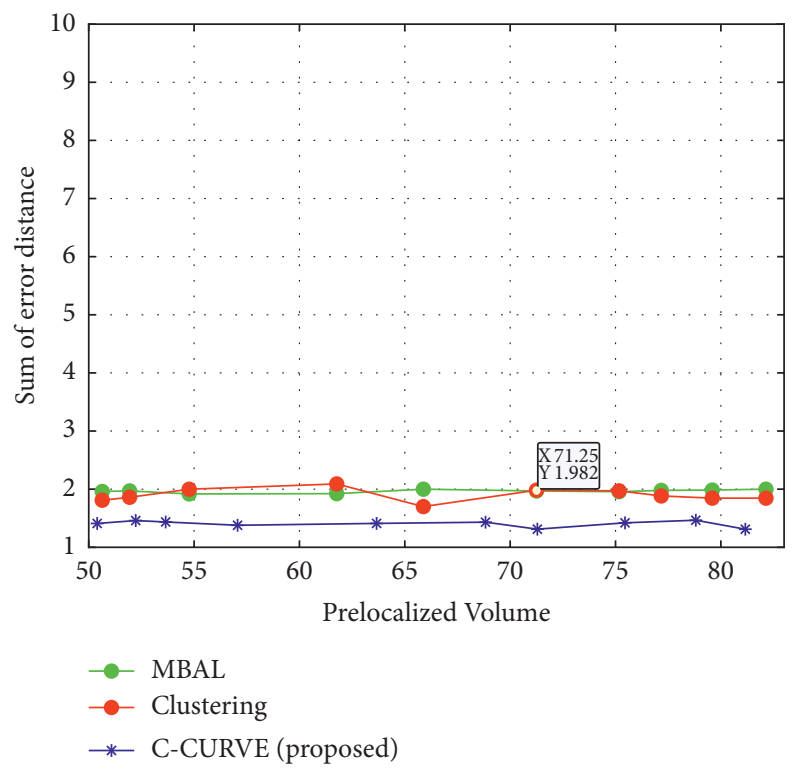

FIGURE 12: Comparison of MBAL, clustering technique, and proposed algorithm.

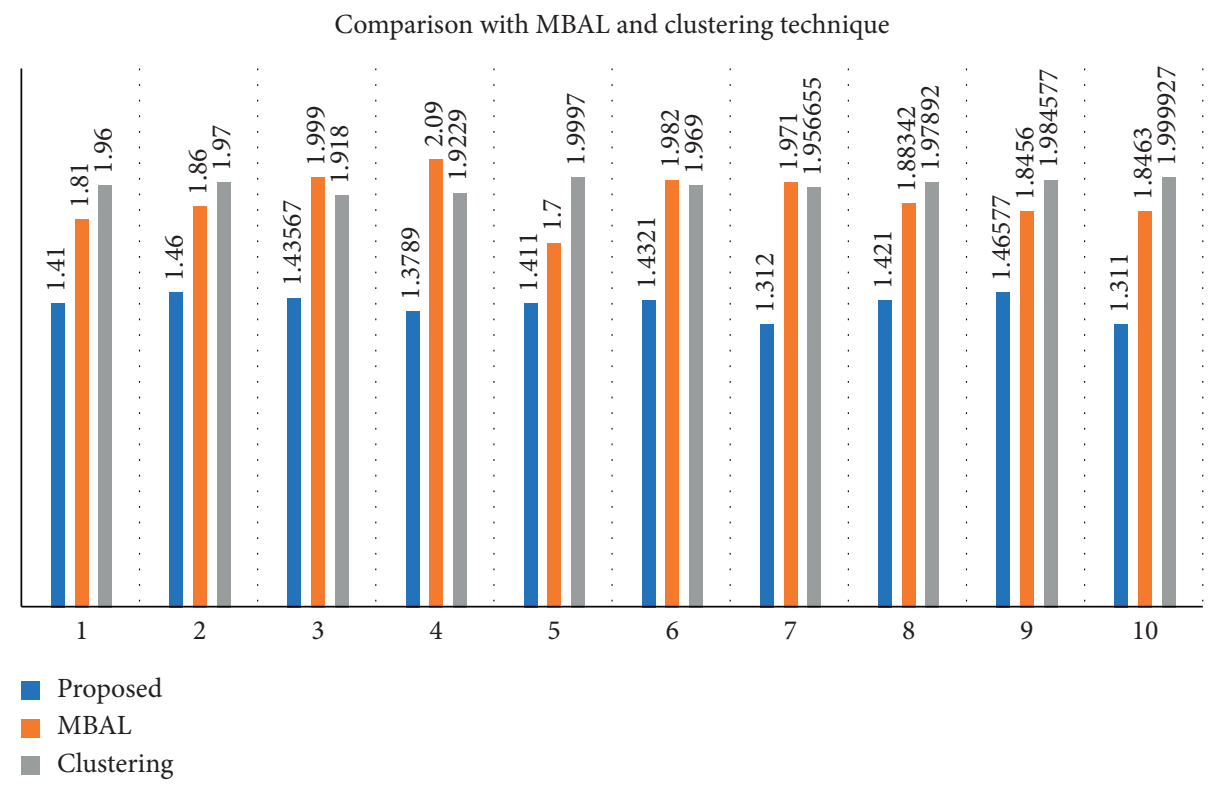

FIGURE 13: Comparison of MBAL, clustering technique, and proposed algorithm with 50\% anchor nodes density.

mobile beacons, and used a fixed trajectory rather than a random walk over the network region. The number of messages broadcast by a beacon node and the path length is used as the main parameter in this scheme. So, if we increased the number of anchor nodes, the number of transmitted messages also increased. For 25 anchor nodes, a movement length of $\sim 602 \mathrm{~m}$ has been covered. In our proposed algorithm, the path is a C-shaped random curve which minimizes the length of random walk for an anchor node which helps to minimize the computation cost and node battery lasts for a longer period. In case of increase in number of anchor nodes in MBAL scheme shown very good performance and decreases $93.3 \%$ of transmitted becaons messages and $95.3 \%$ of movement length (trajectory). However, the network coverage is a limitation of this scheme. With a $50 \%$ of anchor node density and a fixed distance of $70 \mathrm{~m}$, we observed that, over the selected region, the error of MBAL is still very high as compared to our proposed algorithm as shown in Figure 12. The only reason behind this is that the MBAL only focused on a number of broadcast messages in the presence of fewer flying anchors. The proposed scheme has also been compared with the recent techniques proposed in [36], which form a clustering head as compared to triangles in a region. The bounded clusters are always equipped with anchor nodes over the deployed region. In case the region is very huge, the outer 
anchor nodes failed to localize the inner most nodes. Therefore, our technique is far better than the clustering mechanism as shown in Figure 13.

\section{Conclusions and Future Work}

In this work, our focus was to localize the sensor nodes with the help of mobile anchor nodes. To do so, we have proposed a mobile anchor-based localization algorithm that follows the C-shaped trajectories in a 3D-based network and computed the localization error. The mobile anchor node keeps sending the beacons and obtains RSSI values against each node. The successive LQI value of a node can help to identify the average signal strength, which helps to accurately measure the distance. The distance matrix is then formed for triangulation computation, which accurately measures the node position. The simulation result shows that the C-CURVE algorithm shows much efficiency even in case of multipath fading. Our proposed algorithm also provides high accuracy even in presence of noise, due to the use of successive LQI values.

For future work, we are still working on localization error computation in presence of noise. In the second phase of the study, we are computing the complexity of the algorithm and measuring the noise factor in presence of Gaussian and intelligent noise. The noise factor is then eliminated by using extended Kalman filtering. We also compute the lower bound error to sum up this study.

\section{Data Availability}

The RSSI dataset is being taken from pspachos GitHub.

\section{Conflicts of Interest}

The authors declare that there are no conflicts of interest regarding the publication of this paper.

\section{Acknowledgments}

This work was fully supported by the National Nature Science Foundation of China under projects 266343 61673079, and 61703068 and the Natural Science Foundation of Chongqing under project cstc2018jcyjAX0160.

\section{References}

[1] T. Ahmad, X. Li, and B. C. Seet, "Parametric loop division for $3 \mathrm{~d}$ localization in wireless sensor networks," Sensors, vol. 17, no. 7, p. 1697, 2017.

[2] F. Xu, H. Ye, F. Yang, and C. Zhao, "Software defined missioncritical wireless sensor network: architecture and edge offloading strategy," IEEE Access, vol. 7, pp. 10383-10391, 2019.

[3] R. E. Mohamed, A. I. Saleh, M. Abdelrazzak, and A. S. Samra, "Survey on wireless sensor network applications and energy efficient routing protocols," Wireless Personal Communications, vol. 101, no. 2, pp. 1019-1055, 2018.

[4] H. Wu, Z. Ding, and J. Cao, "GROLO: realistic range-based localization for mobile IoTs through global rigidity," IEEE Internet of Things Journal, vol. 6, no. 3, pp. 5048-5057, 2019.
[5] T. Ahmad, X. J. Li, and B. C. Seet, "A self-calibrated centroid localization algorithm for indoor ZigBee WSNs," in Proceedings of the 2016 8th IEEE International Conference on Communication Software and Networks (ICCSN), pp. 455461, Beijing, China, 2016 June.

[6] G. Han, J. Jiang, C. Zhang, T. Q. Duong, M. Guizani, and G. K. Karagiannidis, "A survey on mobile anchor node assisted localization in wireless sensor networks," IEEE Communications Survey, vol. 8, no. 13, pp. 2220-2243.

[7] A. Kumar and R. K. Paul, "Progressive localization using mobile anchor in wireless sensor network," International Journal of Engineering and Computer Science, vol. 6, 4 pages, 2019.

[8] T. Alhmiedat, A. Abu Taleb, and M. Bsoul, "A study on threads detection and tracking systems for military applications using WSNs," International Journal of Computer Applications, vol. 40, no. 15, pp. 12-18, 2012.

[9] M. A. Hussain and K. kyung Sup, "WSN research activities for military application," in Proceedings of the 2009 11th International Conference on Advanced Communication Technology, pp. 271-274, IEEE, Phoenix Park,South Korea, 2009, February.

[10] J. Fan, T. Liang, T. Wang, and J. Liu, "Identification and localization of the jammer in wireless sensor networks," The Computer Journal, vol. 62, no. 10, pp. 1515-1527, 2019.

[11] K. Chintalapudi, T. Fu, J. Paek et al., "Monitoring civil structures with a wireless sensor network," IEEE Internet Computing, vol. 10, no. 2, pp. 26-34, 2006.

[12] J. Masri, R. Szewczyk, A. Mainwaring, D. Culler, and J. Anderson, "Analysis of wireless sensor networks for habitat monitoring," in Proceedings of the Wireless sensor networks, pp. 399-423, Springer, Boston, MA, USA, January 2004.

[13] K. Sha, W. Shi, and O. Watkins, "Using wireless sensor networks for fire rescue applications: requirements and challenges," in Proceedings of the 2006 IEEE International Conference on Electro/Information Technology, pp. 239-244, IEEE, East Lansing, MI, USA, 2006 May.

[14] Y. Li, Z. Wang, and Y. Song, "Wireless sensor network design for wildfire monitoring," in Proceedings of the 2006 6th World Congress on Intelligent Control and Automation, vol. 1, pp. 109-113, IEEE, Dalian, China, 2006 June.

[15] H. Yan, Y. Xu, and M. Gidlund, "Experimental e-health applications in wireless sensor networks," in Proceedings of the 2009 WRI International Conference on Communications and Mobile Computing, vol. 1, pp. 563-567, IEEE, Yunnan, China, 2009 January.

[16] S. Pirbhulal, H. Zhang, M. E Alahi et al., "A novel secure IoTbased smart home automation system using a wireless sensor network," Sensors, vol. 17, no. 1, p. 69, 2017.

[17] S. Elango and P. Gupta, "RSSI based indoor position monitoring using WSN in a home automation application," Acta Electrotechnica et Informatica, vol. 11, no. 4, p. 14, 2011.

[18] A. Boukerche, H. A. B. F. Oliveira, E. F. Nakamura, and A. A. F. Loureiro, "Vehicular ad hoc networks: a new challenge for localization-based systems," Computer Communications, vol. 31, no. 12, pp. 2838-2849, 2008.

[19] T. Ahmad, X. J. Li, and B. C. Seet, “3D localization using social network analysis for wireless sensor networks," in Proceedings of the 2018 IEEE 3rd International Conference on Communication and Information Systems (ICCIS), pp. 88-92, Singapore, Singapore, 2018, December.

[20] T. Ahmad, X. J. Li, and B.-C. Seet, "Noise reduction scheme for parametric loop division $3 \mathrm{D}$ wireless localization 
algorithm based on extended kalman filtering," Journal of Sensor and Actuator Networks, vol. 8, no. 2, p. 24, 2019.

[21] Y. Wang, X. Ma, and G. Leus, "Robust time-based localization for asynchronous networks," IEEE Transactions on Signal Processing, vol. 59, no. 9, pp. 4397-4410, 2011.

[22] Y. S. Lee, J. W. Park, and L. Barolli, "A localization algorithm based on AOA for ad-hoc sensor networks," Mobile Information Systems, vol. 8, no. 1, pp. 61-72, 2012.

[23] P. Singh and S. Agrawal, "TDOA based node localization in WSN using neural networks," in Proceedings of the 2013 International Conference on Communication Systems and Network Technologies, pp. 400-404, IEEE, Gwalior, India, 2013 April.

[24] C. Alippi and G. Vanini, "A RSSI-based and calibrated centralized localization technique for wireless sensor networks," in Proceedings of the Fourth Annual IEEE International Conference on Pervasive Computing and Communications Workshops (PERCOMW'06), p. 5, Pisa, Italy, 2006 March.

[25] D. Koutsonikolas, S. M. Das, and Y. C. Hu, "Path planning of mobile landmarks for localization in wireless sensor networks," Computer Communications, vol. 30, no. 13, pp. 2577-2592, 2007.

[26] Z. Hu, D. Gu, Z. Song, and H. Li, "Localization in wireless sensor networks using a mobile anchor node," in Proceedings of the IEEE/ASME International Conference on Advanced Intelligent Mechatronics (AIM '08), pp. 602-607, Xian, China, July 2008.

[27] H. Cui, Y. Wang, and J. Lv, "Path planning of mobile anchor in three-dimensional wireless sensor networks for localization," Journal of Information and Computational Science, vol. 9, no. 8, pp. 2203-2210, 2012.

[28] H. Li, J. Wang, X. Li, and H. Ma, "Real-time path planning of mobile anchor node in localization for wireless sensor networks," in Proceedings of the IEEE International Conference on Information and Automation (ICIA '08), pp. 384-389, Changsha, China, June 2008.

[29] H. Haddadi, M. Rio, G. Iannaccone, A. Moore, and R. Mortier, "Network topologies: inference, modeling, and generation," IEEE Communications Surveys \& Tutorials, vol. 10, no. 2, pp. 48-69, 2008.

[30] S. Sadowski and P. Spachos, "Rssi-based indoor localization with the internet of things," IEEE Access, vol. 6, pp. 3014930161, 2018.

[31] ZigBee Standards Organisation, ZigBee Specification, ZigBee Alliance, San Ramon, CA, USA, 2008.

[32] T. He, C. Huang, B. M. Blum, J. A. Stankovic, and T. Abdelzaher, "Range-free localization schemes for large scale sensor networks," in Proceedings of the 9th annual international conference on Mobile computing and networking, pp. 81-95, ACM, Los Angeles, CA, USA, September 2003.

[33] T. Ahmad and J. Xue, B.-C. Seet, "3D localization based on parametric loop division and subdivision surfaces for wireless sensor networks," in Proceedings of the 2016 25th Wireless and Optical Communication Conference (WOCC), pp. 1-6, IEEE, New york, NJ, USA, May 2016.

[34] T. Ahmad and J. Xue, W. Jiang and A. Ghaffar, "Frugal sensing: a novel approach of mobile sensor network localization based on fuzzy-logic," in Proceedings of the ACM MobiArch 2020 The 15th Workshop on Mobility in the Evolving Internet Architecture, pp. 8-15, Los Angeles, CA, USA, September 2020.

[35] K. Kim and W. Lee, "MBAL: a mobile beacon-assisted localization scheme for wireless sensor networks," in Proceedings of the 2007 16th International Conference on
Computer Communications and Networks, pp. 57-62, IEEE, Honolulu, HI, USA, August 2007.

[36] S. Sahana and K. Singh, "Cluster based localization scheme with backup node in underwater wireless sensor network," Wireless Personal Communications, vol. 110, no. 4, pp. 1693-1706, 2020. 